\title{
Utilidad de la glicemia y gases venosos centrales como marcador pronóstico en cirugía cardiaca programada
}

\section{Glycemia and central venous blood gas as prognostic markers undergoing cardiac surgery}

Jorge Vivas-Isaza1, Ángel Paternina-Caicedo', Rubén Teheran', Liseth Santoya-Espinosa1, Lina Ortega-Nieves¹, José Rojas-Suárez ${ }^{1}$

\begin{abstract}
Introducction: There is a correlation between supply and demand imbalance of oxygen at the tissue level with central venous blood gas parameters in blood in cardiac surgery patients, however current evidence is inconclusive. Objective: To evaluate the usefulness of venous gases as prognostic marker of major postoperative complications in cardiac surgery patients. Methods: Analytical observational prospective cohort study in patients undergoing cardiac surgery scheduled, admitted to ICU, $\geq 18$ years. Postoperative venous repeated measurements were performed gases. Results: 55 patients were included in this study. There were no baseline demographic differences between patients who developed major complications (CM) and those without (NCM). CM patients had longer times of CPB and aortic clamping, need for blood products, vasoactive and reoperations in ICU than NCM group. PH values at admission to ICU $<7.315$, ScvO2 $<67 \%$ at admission and $<65 \%$ at 6 hours after admission to ICU identify patients who may have adverse events during the 30 days after surgery. Conclusions: pH levels at admission, central venous saturation ICU admission and 6 hours after surgery and blood sugar immediately after cardiac surgery are independent prognostic markers for major complications, including death.
\end{abstract}

\section{RESUMEN}

Introducción: Existe una correlación entre el desbalance del aporte y de-

\section{Key words:}

Blood gas analysis, hydrogen ion concentration, blood glucose, lactic acid, thoracic surgery, postoperative care, postoperative complications, mortality

Hospital Universitario Fundación Favaloro. Buenos Aires, Argentina.

Fecha de recepción: 25 de mayo de 2018

Fecha de aceptación: 23 de noviembre de 2018

\section{ORCID}

https://orcid.org/0000- 0002-1009-0009

Correspondencia:

Jorge Vivas-Isaza

jorge_d_vivas@hotmail.com 
manda de oxígeno a nivel tisular con los parámetros gasométricos en sangre venosa central en pacientes de cirugía cardiaca, sin embargo, la evidencia actual es inconcluyente. Objetivos: Evaluar la utilidad de los gases venosos como marcador pronóstico de complicaciones postoperatorias mayores en pacientes de cirugía cardiaca. Métodos: Estudio observacional analítico de cohortes prospectivo en pacientes sometidos a cirugía cardiaca programada, que ingresaron a $\mathrm{UCl}, \geq 18$ años. Mediciones repetidas postoperatorias de gases venosos fueron realizadas. Resultados: 55 pacientes fueron incluidos en este estudio. No hubo diferencias basales demográficas entre los pacientes que desarrollaron complicaciones mayores (CM) y los que no (nCM). Pacientes CM tuvieron mayores tiempos de CEC y clampeo aortico, necesidad de hemoderivados, vasoactivos y reintervenciones en $\mathrm{UCl}$ que el grupo nCM. Valores de $\mathrm{pH}$ al ingreso a $\mathrm{UCl}<7.315, \mathrm{SvCO}_{2}<67 \%$ al ingreso y $<65 \%$ a las 6 horas después del ingreso a UCI identifican pacientes que pueden tener eventos adversos durante los 30 días postoperatorios. Conclusiones: Los niveles de $\mathrm{pH}$ al ingreso, la saturación venosa central al ingreso a UCI y a las 6 horas después de la cirugía y la glicemia inmediatamente después de la cirugía cardiaca, son marcadores pronósticos independientes para complicaciones mayores, incluyendo muerte.

\section{Palabras clave:}

Análisis de los gases la sangre, concentración de iones de hidrógeno, glucemia, ácido láctico

\section{Introducción}

os gases sanguíneos venosos son considerados el espejo del estado metabólico de la célula. Una deficiente entrega de oxigeno a los tejidos o un aumento en la tasa metabólica tisular, se traducen en una mayor tasa de extracción de oxígeno provocando descenso en los niveles de saturación venosa (SvO2) [1]; mecanismos fisiológicos compensatorios intentarán contrarrestar la alteración, que de persistir, la célula entrará en hipoxia y su metabolismo se tornará anaeróbico, provocando una menor utilización de los sustratos energéticos y la producción de lactato, que afectarán directamente su entorno ácido-base[2],[3].

Este fundamento establece una correlación entre el aporte y la demanda de oxígeno a nivel tisular, y ha sido la base para que el monitoreo de parámetros gasométricos en sangre venosa central como la $\mathrm{SvcO}_{2}$, los niveles de lactato sérico (Lac), el pH y la glicemia, sean utilizados en la práctica clínica como metas para orientar la reanimación en pacientes con estados de hipoperfusión como el choque séptico y trauma craneoencefálico[4]-[8].

Varios estudios han intentado demostrar la utilidad de estas sustancias biológicas en el ámbito perioperatorio[9]-[11], como indicadores del estado metabólico tisular, con el fin de optimizar el aporte de oxígeno, manteniendo un adecuado volumen y tono vascular con terapia hídrica y farmacológica, minimizando el riesgo de transfusión de hemoderivados y sobrecarga hídrica, factores que podrían jugar un papel importante en la morbimortalidad postoperatoria de estos pacientes[12]; sin embargo, los resultados han sido ambiguos, Gattinoni y colaboradores, estudiaron en 10.726 pacientes críticos, los valores de $\mathrm{SvO}_{2}(\geq$ $70 \%$ ) como meta hemodinámica para disminuir la morbimortalidad en estos pacientes, obteniendo resultados negativos[13]. Ranucci et al.[14], demostró en pacientes pediátricos con cardiopatías congénitas que valores de $\mathrm{SvO}_{2}<68 \%$ y lactato sérico $>3 \mathrm{mmol} / \mathrm{l}$ son asociados con pobres resultados postoperatorios. Futier et al.[15], confirman estos datos en pacientes sometidos a cirugía abdominal de alto riesgo quirúrgico, agregando el uso de la diferencia venosa-arterial de $\mathrm{CO}_{2}$ (Delta $\mathrm{CO}_{2}$ ) como herramienta complementaria para identificar pacientes con parámetros hemodinámicos subóptimos durante la terapia guiada por metas.

Ante la evidencia actual inconcluyente, el objetivo de este estudio fue evaluar la utilidad de los gases venosos como marcador pronostico de complicaciones postoperatorias en pacientes sometidos a cirugía cardiaca programada.

\section{Materiales y Métodos}

Se realizó un estudio observacional analítico de cohortes prospectivo de pacientes que fueron sometidos a cirugía cardiaca electiva. Previo al procedimiento, se identificaron los posibles candidatos y a cada uno se le solicitó autorización para participar en el es- 
tudio. Una vez éste es obtenido, se registraron datos generales y clínicos como: edad, sexo, comorbilidades y clase funcional actual. Inmediatamente después de realizada la cirugía, el paciente ingresó a la Unidad de Cuidados Intensivos ( $\mathrm{UCI}$ ), se tomaron muestras de sangre venosa de catéter venoso central al ingreso, a las 2 horas, 6 horas, 12 horas y 24 horas postoperatorias, las cuales se utilizan para medir $\mathrm{pH}$ venoso $(\mathrm{pHv})$, glicemia venosa (Glicv), saturación venosa de oxígeno $\left(\mathrm{SvCO}_{2}\right)$ y lactato sérico (Lact). Asimismo, se registraron datos del período Intraoperatorio acerca de requerimiento de inotrópicos, tiempo de clampeo aórtico y tiempo de circulación extracorpórea. El seguimiento se realizó durante la estancia en UCI, Unidad de Cuidados Intermedios, hospitalización y estancia en domicilio hasta los 45 días postoperatorios. Un resultado compuesto de complicaciones mayores fue usado y definido como una combinación de toda causa de mortalidad en los 30 días y morbilidad mayor (mediastinitis, necesidad de reintervención quirúrgica cardiaca, ventilación mecánica > 7 días, infarto agudo al miocardio y parada cardiaca durante estancia en $\mathrm{UCI}$ ).

Los pacientes fueron divididos en 2 grupos: Grupo A: sin complicaciones mayores; Grupo B: con complicaciones mayores. Los 2 grupos fueron analizados según las características basales, el tipo de cirugía cardiaca realizada y el comportamiento Intraoperatorio. Se analizaron las diferencias de las variables categóricas utilizando $\chi^{2}$. Para las variables continuas, se midió el tipo de distribución utilizando el test de Shapiro-Wilk, aquellas con distribución no paramétrica se analizaron con el test de Mann-Whitney. Las variables categóricas se expresaron con valores absolutos y porcentajes, mientras que las continuas con mediana (rango IQ) o media \pm SD según su distribución. En las variables de exposición, se realizó el análisis de sus diferentes valores en el tiempo usando el test de mediciones repetidas Anova. Un análisis de regresión logística fue usado para identificar la asociación de riesgo independiente para complicaciones mayores postoperatorias. Con el fin de obtener los valores para los puntos de corte asociados a los resultados, se utilizó el análisis de curva ROC (receiver operator characteristic). El análisis fue desarrollado con los programas: GraphPad Prism versión 6.0 y Stata versión 13.1.

\section{Resultados}

Desde septiembre de 2012 a septiembre de 2013, se realizaron 69 cirugías cardiacas, 9 se excluyeron de acuerdo a los criterios de elegibilidad y otros 5 procedimientos correspondieron a reintervenciones quirúrgicas. De los 55 pacientes (mujeres: 21, hombres: 34 ), al $54,6 \%$ se les realizó cirugía de bypass coronario (CRBC), 25\% cirugía valvular simple y el 16,3\% cirugía valvular asociado a otro procedimiento. Dieciocho pacientes $(32,7 \%)$ tuvieron complicaciones mayores, incluidas 5 muertes $(9,1 \%)$.

La Tabla 1 demuestra las características demográficas de la población, detalla los datos preoperatorios e intraoperatorios y la diferencia entre los grupos. La mediana de edad fue de $58(51,5-65,5)$ y $59,5(52,7-$ $67,2)$ años en el grupo sin complicaciones mayores (nCM) y con complicaciones mayores (CM) respectivamente. El sexo masculino predominó sin diferencia significativa en ambos grupos $(64,75$ vs $66,7 \%, p=$ 0,88 ). La prevalencia de comorbilidades como hipertensión arterial, diabetes mellitus, insuficiencia renal crónica y pobre fracción de eyección ventricular (< $45 \%$ ) fue similar en ambos grupos. Los pacientes a los que se les realizaron cirugías cardiacas complejas presentaron en mayor número complicaciones mayores que aquellos a los cuales se les realizaron cirugías menos complejas (38,8\% vs 5,5\%; $p<0,05)$.

Los pacientes con complicaciones mayores (CM) tuvieron tiempos de clampeo aórtico (59,5 min vs 34 min; $p<0,05)$ y circulación extracorpórea (80 min vs 45 min; $p<0,05$ ) significativamente mayores que los del grupo que no presentaron complicaciones mayores (noCM). De igual forma, éstos tuvieron mayor necesidad de transfusión de hemoderivados $(77,7 \%$ vs $10,8 \% ; p<0,05)$, uso de drogas vasoactivas en $\mathrm{UCl}$ $(83,3 \%$ vs $51,3 \% ; p<0,05)$ y duraron más tiempo en ventilación mecánica $(1,62 \pm 0,69$ días vs $6,44 \pm 9,9$ días; $p<0,05)$. No hubo diferencia significativa entre los grupos con respecto a la presencia de infección del sitio operatorio (Tabla 2).

El grupo de pacientes que no desarrolló complicaciones mayores ( $\mathrm{nCM}$ ) tuvieron al ingreso a la UCI niveles más alto de $\mathrm{pH}(7,29 \pm 0,06$ vs 7,27 \pm $0,06 ; p=0,238)$ y saturación venosa central $(68 \pm$ 11 vs $60 \pm 14 ; p=0,059)$ que aquellos que tuvieron complicaciones mayores, sin embargo, la diferencia no fue significativa hasta que fue ajustada con el análisis multivariado de regresión logística ( $P$ ajustado $=0,040$ y $P$ ajustado $=0,028$, respectivamente). De igual forma, los niveles de glicemia después de la admisión a UCI y la saturación venosa central a las 6 $h$, fueron más altos en el grupo noCM que en el de CM $[(139 \pm 30$ vs $111 \pm 27 ; p<0,05)$ y $(59 \pm 8$ vs 53 $\pm 12 ; p<0,05)$ respectivamente], diferencia que se mantuvo al ajuste multivariado. El comportamiento del lactato sérico en ambos grupos fue similar, sin di- 


\begin{tabular}{|c|c|c|c|c|}
\hline Variables & $\begin{array}{l}\text { Población total } \\
n=55(\%)\end{array}$ & $\begin{array}{l}\text { No complicación } \\
\text { mayor }(n=37)\end{array}$ & $\begin{array}{c}\text { complicación } \\
\text { mayor } \\
(n=18)\end{array}$ & $\begin{array}{c}\text { Valor de } p \\
*(<0,05)\end{array}$ \\
\hline Edad & $59(52-66)$ & $58(51,5-65,5)$ & $59,5(52,75-67,25)$ & 0,5904 \\
\hline Sexo & $\begin{array}{c}\text { M:34 }(61,8 \%), \\
F: 21(38,2) \%\end{array}$ & $\begin{array}{c}\text { M:22 }(64,7 \%) \\
\text { F:12 }(32,3 \%)\end{array}$ & $\begin{array}{c}\text { M:12 (66,7\%) } \\
\text { F:6 (33,3\%) }\end{array}$ & 0,8876 \\
\hline Clase funcional $<4$ met & $31(56,3 \%)$ & $21(56,7 \%)$ & $10(55,5 \%)$ & 0,9328 \\
\hline \multicolumn{5}{|l|}{ Comorbilidades preoperatorias } \\
\hline Hipertensión arterial & $45(81,8 \%)$ & $28(75,6 \%)$ & $17(94,4 \%)$ & 0,0904 \\
\hline Diabetes mellitus & $20(36,3 \%)$ & $14(37,8 \%)$ & $6(33,3 \%)$ & 0,7445 \\
\hline $\mathrm{FEVI}<45 \%$ & $27(49 \%)$ & $16(43,2 \%)$ & $11(61,1 \%)$ & 0,2136 \\
\hline Insuficiencia renal crónica & $7(12,7 \%)$ & $4(10,8 \%)$ & $3(16,6 \%)$ & 0,5409 \\
\hline EPOC & $4(7,3 \%)$ & $3(8,1 \%)$ & $1(5,5 \%)$ & 0,7323 \\
\hline \multicolumn{5}{|l|}{ Tipo de cirugía } \\
\hline Cirugía cardiaca compleja & $9(16,3 \%)$ & $2(5,5 \%)$ & $7(38,8 \%)$ & $0,0016^{*}$ \\
\hline CRBC & $30(54,6 \%)$ & $22(59,4 \%)$ & $8(44,4 \%)$ & \\
\hline Atrioplastia & $1(1,8 \%)$ & $1(2,7 \%)$ & 0 & \\
\hline RVA & $8(14,5 \%)$ & $7(18,9 \%)$ & $1(5,5 \%)$ & \\
\hline RVM & $6(10,9 \%)$ & $4(10,8 \%)$ & $2(11,1 \%)$ & \\
\hline$R V M+A$ & $2(3,6 \%)$ & $0(0 \%)$ & $2(11,1 \%)$ & \\
\hline$R V A+R A A+R I C$ & $4(7,3 \%)$ & $0(0 \%)$ & $4(22,2 \%)$ & \\
\hline RVM + MAZE & $1(1,8 \%)$ & $1(2,7 \%)$ & 0 & \\
\hline$R V M+C R B C$ & $1(1,8 \%)$ & $0(0 \%)$ & $1(5,5 \%)$ & \\
\hline VPM & $1(1,8 \%)$ & $1(2,7 \%)$ & 0 & \\
\hline Resección de mixoma + VPM + VPT & $1(1,8 \%)$ & $1(2,7 \%)$ & 0 & \\
\hline \multicolumn{5}{|l|}{ Variables intraoperatorias } \\
\hline Inotrópicos intraoperatorios & $25(45 \%)$ & $14(37,8 \%)$ & $11(61,1 \%)$ & 0,1039 \\
\hline Tiempo de clampeo aórtico (min) & 44,8 & $34(21-56)$ & $59,5(36,5-103)$ & $0,0009 *$ \\
\hline Tiempo de CEC (min) & 72 & $45(35-65)$ & $80(53,75-124,8)$ & $<0,0001^{*}$ \\
\hline
\end{tabular}

\begin{tabular}{lcccc}
\hline \multicolumn{3}{c}{ Tabla 2. Desenlaces } \\
\hline \multicolumn{1}{c}{ Variables } & $\mathbf{n = 5 5 ( \% )}$ & $\begin{array}{c}\text { No complicación } \\
\text { mayor }(\mathbf{n}=\mathbf{3 7})\end{array}$ & $\begin{array}{c}\text { Complicación mayor } \\
(\mathbf{n}=\mathbf{1 8})\end{array}$ & $\begin{array}{c}\text { Valor de } \mathbf{p} \text { *(< } \\
\mathbf{0 , 0 5})\end{array}$ \\
Días con ventilación mecánica & $3,05 \pm 6,27$ & $1,62 \pm 0,69$ & $6,44 \pm 9,9$ & $0,0003^{*}$ \\
Inotrópicos en UCl & $34(61,8 \%)$ & $19(51,3 \%)$ & $15(83,3 \%)$ & $0,0220 *$ \\
Infección del sitio operatorio & $9(16,3 \%)$ & $4(10,8 \%)$ & $5(27,7 \%)$ & 0,1105 \\
Transfusión de hemoderivados & $18(32,7 \%)$ & $4(10,8 \%)$ & $14(77,77 \%)$ & $<0,0001^{*}$ \\
\hline
\end{tabular}

ferencias significativas en las diferentes muestras de gases venosos (Tabla 3 ).

El punto de corte donde los niveles de glicemia al ingreso a $\mathrm{UCl}$ se asociaron a complicaciones mayores fue $<135 \mathrm{mg} / \mathrm{dl}$, producto de un área bajo la curva (AUC) en el análisis de curva ROC de 0,78 (95\% inter- 


\begin{tabular}{|c|c|c|c|c|}
\hline & $\begin{array}{c}\text { Pacientes sin } \\
\text { complicaciones } \\
\text { mayores }\end{array}$ & $\begin{array}{c}\text { Pacientes con } \\
\text { complicaciones } \\
\text { mayores }\end{array}$ & $p$ & p ajustada \\
\hline \multicolumn{5}{|c|}{$\mathrm{pH}$ venoso } \\
\hline Inicial & $7,29 \pm 0,06$ & $7,27 \pm 0,06$ & 0,238 & $0,040 *$ \\
\hline $6 \mathrm{~h}$ & $7,28 \pm 0,05$ & $7,24 \pm 0,07$ & 0,101 & 0,131 \\
\hline $24 \mathrm{~h}$ & $7,32 \pm 0,05$ & $7,28 \pm 0,11$ & 0,100 & 0,144 \\
\hline \multicolumn{5}{|l|}{$\mathrm{SvcO}_{2}$} \\
\hline Inicial & $68 \pm 11$ & $60 \pm 14$ & 0,059 & $0,028 *$ \\
\hline $6 \mathrm{~h}$ & $59 \pm 8$ & $53 \pm 12$ & $0,034^{*}$ & $0,045^{*}$ \\
\hline $24 \mathrm{~h}$ & $58 \pm 11$ & $58 \pm 8$ & 0,992 & 0,935 \\
\hline \multicolumn{5}{|c|}{ Glicemia CVC } \\
\hline Inicial & $139 \pm 30$ & $111 \pm 27$ & $0,007^{*}$ & $0,017^{*}$ \\
\hline $6 \mathrm{~h}$ & $145 \pm 35$ & $148 \pm 38$ & 0,758 & 0,745 \\
\hline $24 \mathrm{~h}$ & $127 \pm 25$ & $151 \pm 63$ & 0,084 & 0,308 \\
\hline \multicolumn{5}{|c|}{ Lactato sérico } \\
\hline Inicial & $2,6 \pm 1,6$ & $2,6 \pm 1,4$ & 0,940 & 0,835 \\
\hline $6 \mathrm{~h}$ & $2,2 \pm 1,3$ & $1,9 \pm 1,0$ & 0,485 & 0,259 \\
\hline $24 \mathrm{~h}$ & $1,7 \pm 1,0$ & $2,1 \pm 1,1$ & 0,186 & 0,823 \\
\hline
\end{tabular}

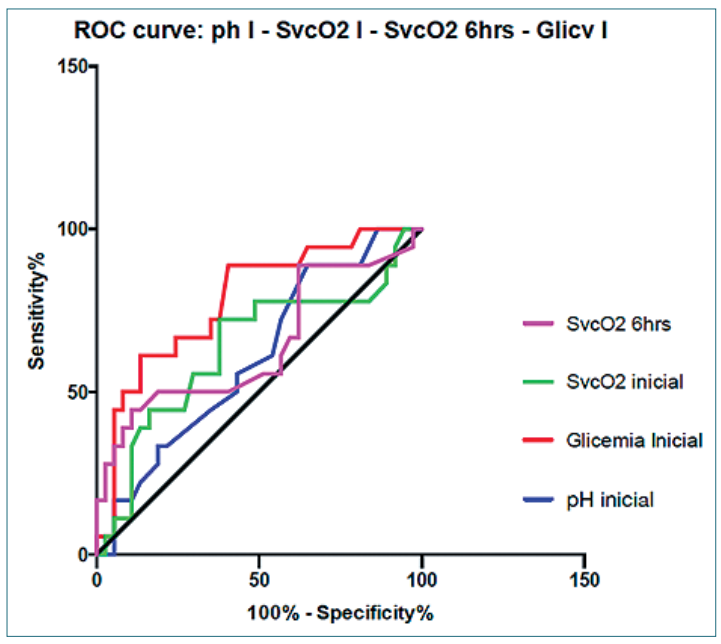

Figura 1. Comparación de curva ROC entre las variables $\mathrm{pH}$ inicial, $\mathrm{SvcO}_{2}$ inicial, $\mathrm{SvcO}_{2} 6 \mathrm{~h}$ y glicemia venosa central inicial.

valo de confianza [IC], 0,65-0,91; $p=0,0008$ ) (Figura 1). Para $\mathrm{pH}$ al ingreso fue $<7.315, \mathrm{AUC}=0,60(95 \%$ IC: $0,44-0,75 ; p=0,21)$. $\mathrm{SvCO}_{2}$ al ingreso fue $<67,5$, $A U C=0,63(95 \%$ IC: $0,46-0,80 ; p=0,11), \mathrm{SvCO}_{2} a$

\begin{tabular}{lcc}
\multicolumn{3}{c}{ Tabla 4. Análisis de curva ROC } \\
\hline \multicolumn{1}{c}{ Variable } & AUC & IC 95\% \\
$\mathrm{pH}$ inicial & 0,60 & $0,44-0,75$ \\
$\mathrm{SvCO}_{2}$ inicial & 0,63 & $0,46-0,80$ \\
$\mathrm{SvCO}_{2}$ 6 h & 0,65 & $0,49-0,82$ \\
Glicemia inicial & $0,78 *$ & $0,65-0,91$ \\
\hline
\end{tabular}

las 6 fue $<65,5, A \cup C=0,64(95 \%$ IC: $0,47-0,81 ; p=$ 0,08) (Tabla 4).

\section{Discusión}

Este estudio demuestra que niveles de glicemia, pH y saturación venosa central, inmediatamente después del ingreso a $\mathrm{UCl}$, se relacionan con complicaciones mayores. Valores de $\mathrm{pH}$ al ingreso a $\mathrm{UCl}<7.315$, $\mathrm{SvCO}_{2}<67 \%$ al ingreso y $<65 \%$ a las 6 horas después del ingreso a UCl identifican pacientes que pueden tener eventos adversos durante los 30 días postoperatorios. Además, niveles de glicemia $<135 \mathrm{mg} / \mathrm{dl}$ al ingreso a UCl, están asociados independientemente con complicaciones mayores postoperatorias, incluida 
muerte, en los pacientes sometidos a cirugía cardiaca.

El uso de los valores de gases venosos para la proyección pronóstica de resultados adversos en el contexto perioperatorio ha sido controversial y aún en la actualidad se mantiene indefinida. La cirugía cardiaca de manera peculiar altera la perfusión tisular desencadenando estados de hipoxia tisular. Estudios recientes ratifican la correlación entre niveles bajos de $\mathrm{SCVO}_{2}$ e hiperlactatemia durante y después de la derivación cardiopulmonar con mortalidad y complicaciones postoperatoria[16],[17], además confirman que la alteración en estos es multifactorial y no puede ser predicha con la medición de parámetros hemodinámicos, sugiriendo entonces su monitoreo clínico solo o en combinación para tratar la hipoperfusión tisular y prevenir el desarrollo de disfunción orgánica[14],[18]. En acuerdo con estos estudios, nuestros resultados confirman la relevancia de la saturación venosa central de oxígeno en los resultados postoperatorios y el impacto de las primeras 6 horas postoperatorias como tiempo decisivo en el pronóstico de los pacientes, momento en el cual se debe hacer uso de las herramientas necesarias para restablecer la relación demanda-aporte de oxígeno tisular[19]. Los valores de lactato sérico en sangre venosa central no fueron asociados con morbimortalidad, sin embargo, los niveles a las 24 horas postoperatorias fue mayor en el grupo con complicaciones mayores, diferencia que no fue significativamente estadística.

Muchos estudios se han realizado en el paciente cardiaco quirúrgico para determinar la repercusión de las alteraciones en los niveles de glicemia. En el paciente diabético y en el no diabético, tanto la hiperglicemia como la hipoglicemia se ha relacionado en eventos adversos como muerte, infecciones severas, duración de estancia hospitalaria, arritmias cardiacas y secuelas neurológicas. Jones et al. [20], demostró en 2.297 pacientes postoperatorios de cirugía de bypass coronario que aquellos que inmediatamente después del procedimiento presentaban niveles de glicemia > $200 \mathrm{mg} / \mathrm{dl}$ tenían un riesgo significativamente mayor de ventilación mecánica prolongada, estancia hospitalaria y mortalidad, comparados con los pacientes con niveles normales de glicemia (80-110 mg/dl). Datos similares encontró Doesnt y colaboradores en pacientes con picos de hiperglicemia $(15 \mathrm{mmol} / \mathrm{l})$ durante el bypass cardiopulmonar (BCP) sugiriendo que los niveles altos de glicemia son un factor independiente de mortalidad en pacientes diabéticos y no diabéticos (OR 1,06; IC 95\% 1,03-1,09)[21].

Como respuesta a estas complicaciones se diseñaron estrategias para tratar la hiperglicemia coligada al BCP con esquemas terapéuticos estrictos con insulina en bolos o infusiones que incluían diferentes umbrales de glicemia para iniciar tratamiento, los cuales lograron una disminución subsecuente de las tasas de morbilidad y mortalidad[22],[23]. Sin embargo, estos esquemas no son inocuos, y someten a los pacientes al riesgo de desarrollar hipoglicemia con sus efectos deletéreos. Stamou et al.[24], en su cohorte prospectiva de 2.538 pacientes cardiacos quirúrgicos con esquema de insulina en infusión para mantener glicemia < $200 \mathrm{mg} / \mathrm{dl}$, comparados con los que se mantuvieron normoglicémicos, aquellos con hipoglicemia secundaria ( $97 \%$ vs 3\%) tuvieron mayor necesidad de transfusiones sanguíneas, tiempo de ventilación mecánica, soporte dialítico, estancia hospitalaria y mayor mortalidad.

Ante la evidencia dispersa con respecto a la mejor forma de realizar el control glicémico, los investigadores del NICE-SUGAR trial brindan al clínico, el soporte científico para realizar el manejo metabólico adecuado del paciente critico-quirúrgico. Comparando 2 esquemas terapéuticos en 6.104 pacientes: intensivo (80-108 mg/dl) vs convencional (< $180 \mathrm{mg} / \mathrm{dl}$ ), logran establecer que los pacientes con esquema intensivo presentaron mayor número de muertes y ésto asociado a más episodios de hipoglicemia severa[25].

Los resultados que arroja nuestro estudio están a favor de las conclusiones del NICE-SUGAR trial. Aunque no tuvimos un esquema terapéutico definido de control glicémico, ni realizamos seguimiento metabólico durante la estancia en $\mathrm{UCl}$, podemos inferir que niveles de glicemia $<135 \mathrm{mg} / \mathrm{dl}$ inmediatamente después del procedimiento quirúrgico se asocian de forma independiente a complicaciones mayores en los pacientes sometidos a cirugía cardiaca programada. No podemos establecer si la glicemia $>180 \mathrm{mg} / \mathrm{dl}$ o la normoglicemia (80-108 mg/dl) son nocivas o inocuas, ni tampoco que la hipoglicemia ( $<60 \mathrm{mg} / \mathrm{dl}$ ) sea el causal de los resultados adversos, sin embargo, nuestra hipótesis es que los pacientes que ingresan a UCI con glicemias limítrofes (cercanas a $140 \mathrm{mg} / \mathrm{dl}$ ) tienen mayor riesgo de presentar episodios de hipoglicemia y con ésta de padecer eventos adversos.

El grupo de los pacientes con complicaciones mayores requirieron en su evolución clínica en $\mathrm{UCl}$ de más días de ventilación mecánica, drogas vasoactivas y transfusión de hemoderivados que el grupo sin complicaciones mayores.

Nuestro estudio tiene las limitaciones de ser realizado en un solo centro, con una pequeña muestra de la población por lo que no se pueden generalizar los resultados, además de las mencionadas con respecto al seguimiento de intervenciones realizadas como parte del tratamiento en $\mathrm{UCI}$. 


\section{Conclusión}

Los niveles de $\mathrm{pH}$ al ingreso, la saturación venosa central al ingreso a $\mathrm{UCl}$ y a las 6 horas después de la cirugía y la glicemia inmediatamente después de la cirugía cardiaca, son marcadores pronósticos independientes para complicaciones mayores, incluyendo muerte. Se necesitan estudios controlados aleatorizados en nuestra población para determinar si estrategias para optimizar la perfusión tisular y el control metabólico pueden mejorar los resultados de los pacientes sometidos a cirugía cardiaca programada.

\section{Referencias}

1. Beest $P$, Wietasch $G$, Scheeren $T$, Spronk $P$, et al. Clinical review: use of venous oxygen saturations as a goal - a yet unfinished puzzle. 2011;15(5):232-41.

2. Shepherd SJ, Pearse RM. Role of central and mixed venous oxygen saturation measurement in perioperative care. Anesthesiology. 2009 Sep;111(3):64956. https://doi.org/10.1097/ ALN.0b013e3181af59aa PMID:19672190

3. Tipping R, Berry R, Nesbitt I. Mechanisms of hypoxaemia and the interpretation of arterial blood gases. Surgery. 2012;30(10):505-12.

4. Rivers E, Nguyen B, Havstad S, Ressler J, Muzzin A, Knoblich B, et al.; Early Goal-Directed Therapy Collaborative Group. Early goal-directed therapy in the treatment of severe sepsis and septic shock. N Engl J Med. 2001 Nov;345(19):1368-77. https:// doi.org/10.1056/NEJMoa010307 PMID:11794169

5. Dellinger RP, Carlet JM, Masur $\mathrm{H}$, Gerlach H, Calandra T, Cohen J, et al.; Surviving Sepsis Campaign Management Guidelines Committee. Surviving Sepsis Campaign guidelines for management of severe sepsis and septic shock. Crit Care Med. 2004 Mar;32(3):858-73. https://doi.org/10.1097/01. CCM.0000117317.18092.E4 PMID:15090974

6. Dellinger R, Levy M, Carlet J, et al. Surviving sepsis campaign guidelines for management of severe sepsis and septic shock. Crit Care Med. 2008;34:17-60. PMID:18158437

7. Dellinger R, Levy $M$, Rhodes $A$, Annane D, Gerlach H, Opal SM, et al. Surviving sepsis campaign guidelines for management of severe sepsis and septic shock. Crit Care Med. 2013;41(2):580637. https://doi.org/10.1097/ CCM.0b013e31827e83af PMID:23353941

8. Di Filippo A, Gonnelli C, Perretta L, Zagli G, Spina R, Chiostri M, et al. Low central venous saturation predicts poor outcome in patients with brain injury after major trauma: a prospective observational study. Scand J Trauma Resusc Emerg Med. 2009 May;17(1):23. https://doi. org/10.1186/1757-7241-17-23 PMID:19460137

9. Pearse R, Dawson D, Fawcett J, Rhodes A, Grounds RM, Bennett ED. Changes in central venous saturation after major surgery, and association with outcome. Crit Care. 2005;9(6):R694-9. https://doi.org/10.1186/cc3888 PMID:16356220

10. Jakob S, Bracht H, Eigenmann V, et al.; Collaborative Study Group on Perioperative ScvO2 Monitoring. Multicentre study on peri- and postoperative central venous oxygen saturation in high-risk surgical patients. Crit Care. 2006;10(6):R158. https://doi.org/10.1186/cc5094 PMID:17101038
11. Silva JM Jr, Oliveira AM, de Morais SZ, de Araújo LS, Victoria LG, Marubayashi LY. Influence of central venous oxygen saturation on in-hospital mortality of surgical patients. Rev Bras Anestesiol. 2010 Nov-Dec;60(6):593-602. https://doi.org/10.1016/ S0034-7094(10)70074-X PMID:21146055

12. Grocott MP, Dushianthan $A$, Hamilton MA, Mythen MG, Harrison D, Rowan K; Optimisation Systematic Review Steering Group. Perioperative increase in global blood flow to explicit defined goals and outcomes following surgery. Cochrane Database Syst Rev. 2012 Nov; 11:CD004082. https:// doi.org/10.1002/14651858. CD004082.pub5 PMID:23152223

13. Gattinoni $L$, Brazzi $L$, Pelosi $P$, Latini R, Tognoni G, Pesenti A, et al. A trial of goal-oriented hemodynamic therapy in critically ill patients. SvO2 Collaborative Group. N Engl J Med. 1995 Oct;333(16):102532. https://doi.org/10.1056/ NEJM199510193331601 PMID:7675044

14. Ranucci $M$, Isgrò $G$, Carlucci C, De La Torre T, Enginoli S, Frigiola A; Surgical and Clinical Outcome REsearch Group. Central venous oxygen saturation and blood lactate levels during cardiopulmonary bypass are associated with outcome after pediatric cardiac surgery. Crit Care. 2010;14(4):R149. 
PMID:20684758

15. Futier $E$, Robin $E$, Jabaudon $M$, Guerin R, Petit A, Bazin JE, et al. Central venous $\mathrm{O}_{2}$ saturation and venous-to-arterial $\mathrm{CO}_{2}$ difference as complementary tools for goal-directed therapy during high-risk surgery. Crit Care. 2010;14(5):R193. https://doi.org/10.1186/cc9310 PMID:21034476

16. Svenmarker $S$, Häggmark $S$, Östman $\mathrm{M}$, Holmgren $\mathrm{A}$, Näslund $U$. Central venous oxygen saturation during cardiopulmonary bypass predicts 3-year survival. Interact Cardiovasc Thorac Surg. 2013 Jan;16(1):21-6. https:// doi.org/10.1093/icvts/ivs363 PMID:23065747

17. Hajjar LA, Almeida JP, Fukushima JT, Rhodes A, Vincent JL, Osawa EA, et al. High lactate levels are predictors of major complications after cardiac surgery. J Thorac Cardiovasc Surg. 2013 Aug;146(2):455-60. https://doi. org/10.1016/j.jtcvs.2013.02.003 PMID:23507124

18. Hu BY, Laine GA, Wang $S$, Solis RT. Combined central venous oxygen saturation and lactate as markers of occult hypoperfusion and outcome following cardiac surgery. J Cardiothorac Vasc Anesth. 2012
Feb;26(1):52-7. https://doi. org/10.1053/j.jvca.2011.07.021 PMID:21924630

19. Litton E, Silbert B, Ho KM. Clinical predictors of a low central venous oxygen saturation after major surgery: a prospective prevalence study. Anaesth Intensive Care. 2015 Jan;43(1):59-65. https://doi.g/10.1177/031005 7X1504300109 PMID:25579290

20. Jones KW, Cain AS, Mitchell JH, Millar RC, Rimmasch HL, French $T K$, et al. Hyperglycemia predicts mortality after CABG: postoperative hyperglycemia predicts dramatic increases in mortality after coronary artery bypass graft surgery. J Diabetes Complications. 2008 Nov-Dec;22(6):365-70. https://doi.org/10.1016/j. jdiacomp.2007.05.006 PMID:18413193

21. Doenst $T$, Wijeysundera $D$, Karkouti K, Zechner C, Maganti $M$, Rao V, et al. Hyperglycemia during cardiopulmonary bypass is an independent risk factor for mortality in patients undergoing cardiac surgery. J Thorac Cardiovasc Surg. 2005 Oct;130(4):1144. https://doi. org/10.1016/j.jtcvs.2005.05.049 PMID:16214532

22. van den Berghe $G$, Wouters $P$, Weekers F, Verwaest C, Bruy- ninckx $F$, Schetz $M$, et al. Intensive insulin therapy in critically ill patients. N Engl J Med. 2001 Nov;345(19):1359-67. https:// doi.org/10.1056/NEJMoa011300 PMID:11794168

23. Furnary AP, Gao G, Grunkemeier GL, Wu Y, Zerr KJ, Bookin SO, et al. Continuous insulin infusion reduces mortality in patients with diabetes undergoing coronary artery bypass grafting. J Thorac Cardiovasc Surg. 2003 May;125(5):1007-21. https:// doi.org/10.1067/mtc.2003.181 PMID:12771873

24. Stamou SC, Nussbaum M, Carew JD, Dunn K, Skipper E, Robicsek F, et al. Hypoglycemia with intensive insulin therapy after cardiac surgery: predisposing factors and association with mortality. J Thorac Cardiovasc Surg. 2011 Jul;142(1):166-73. https://doi. org/10.1016/j.jtcvs.2010.09.064 PMID:21397274

25. Finfer S, Chittock DR, Su SY, Blair D, Foster D, Dhingra $V$, et al.; NICE-SUGAR Study Investigators. Intensive versus conventional glucose control in critically ill patients. N Engl J Med. 2009 Mar;360(13):128397. https://doi.org/10.1056/NEJMoa0810625 PMID:19318384 